\title{
RELATIVISTIC EFFECTS ON THE CONTRIBUTION OF THE LOCAL-SPIN-DENSITY CORRELATION ENERGY TO IONIZATION POTENTIALS
}

\author{
A. SAVIN. P. SCHWERDTFEGER, H. PREUSS, H. SILBERBACH and H. STOLL \\ Insthut für Thicoretische Chemie der Unin ersität Stuttgart. \\ P'faffichnaldring 55. D.7000 Stuttgart So. Federal Republic of German
}

Received 6 Aprll 1983: in final form 18 April 1983

\begin{abstract}
The contribution of the local-spin-density correlation energy to the ionization potential of some group IA, IB, IIA and IIB one-halence-electron atoms has been calculated; relaxation of the core orbitals lowers it by 10-20\%; relativistic effects enhance it by up to $30 \%$.
\end{abstract}

In order to see to what extent relativistic effects modify the correlation energy we decided to study the contribution of the correlation energy to the ionization potential of some one-valence-electron systems (K, Rb, Cs. Cu, Ag. Au, $\mathrm{Ca}^{+}, \mathrm{Sr}^{+}, \mathrm{Ba}^{+}, \mathrm{Zn}^{+}, \mathrm{Cd}^{+}, \mathrm{Hg}^{+}$). In contrast to Migdalek and Baylis [1] who considered the influence of core-valence correlation only, we included the core-correlation as well.

We did separate calculations for each atom and the corresponding ion, both at the Hartree-Fock (HF) and the Dirac-Fock (DF) level, by using the program RELAMC of Desclaux [2]. (The HF results were obtained by changing the velocity of light to $10^{10} \mathrm{au}$.) We defined the spin up and spin down densities $\left(\rho_{+}\right.$ and $\rho_{-}$, respectively) by using the orbital densities produced by RELAMC: $\rho_{-}$as one half of the core density, $\rho_{+}$as the sum of $\rho_{-}$and the valence density.

These densities were used in the calculation of the correlation energy in the local-spin-density (LSD) approximation. Without self-interaction correction it is given by

$E_{\mathrm{c}}\left[\rho_{+}, \rho_{-}\right]=\int\left(\rho_{+}+\rho_{-}\right) \epsilon_{\mathrm{c}}\left(\rho_{+}, \rho_{-}\right) \mathrm{d} r$.

$\epsilon_{\mathrm{c}}\left(\rho_{+}, \rho_{-}\right)$is the correlation energy per particle of the homogeneous electron gas with partial densities $\rho_{+}$ and $\rho_{-}$.

Two types of calculations were made for the correlation energy: in the first one the non-relativistic param- eterization of $\epsilon_{\mathrm{c}}$ by Vosko et al. [3] was used; in the second a scaling factor was introduced for $\epsilon_{\mathrm{c}}$. This density-dependent scaling factor is given by the ratio of the relativistic $\epsilon_{\mathrm{c}}\left(\rho_{+}+\rho_{-}\right)$of Ramana and Rajagopal [4] and the corresponding non-relativistic value of von Barth and Hedin [5].

We introduced a self-interaction correction (SIC) into $E_{\mathrm{c}}$ by using the definition of Stoll, Pavlidou and Preuss (SPP SIC) [6]:

$E_{\mathrm{c}}^{\prime}\left[\rho_{+}, \rho_{-}\right]=E_{\mathrm{c}}\left[\rho_{+} \rho_{-}\right]-E_{\mathrm{c}}\left[\rho_{+}, 0\right]-E_{\mathrm{c}}\left[0, \rho_{-}\right]$.

An alternative SIC is that of Perdew and Zunger (PZ SIC) [7].

In the frozen-core approximation the contribution of the correlation energy (without SIC) to the ionization potential is given by:

$\Delta E_{\mathrm{c}}=E_{\mathrm{c}}\left[\rho_{+}, \rho_{-}\right]-E_{\mathrm{c}}\left[\rho_{-}, \rho_{-}\right]$.

By using (2) the corresponding value for SPP SIC is obtained:

$\Delta E_{\mathrm{c}}^{\prime}=\Delta E_{\mathrm{c}}-\left(E_{\mathrm{c}}\left[\rho_{+}, 0\right]-E_{\mathrm{c}}\left[0, \rho_{-}\right]\right)$,

while with the SIC of Perdew and Zunger this is:

$\Delta E_{\mathrm{c}}^{\prime \prime}=\Delta E_{\mathrm{c}}-E_{\mathrm{c}}\left[\rho_{+}-\rho_{-}, 0\right]$.

We did not take into account the effect of the correlation potential on $\rho_{+}$and $\rho_{-}$. In our experience $[8,9]$ the resulting energetic effects are small (e.g. 0.4 mhartree for $K[9]$ ). 
Table 1

Contributions to the ionization potential, IP (in mhartree)

\begin{tabular}{|c|c|c|c|c|c|c|c|c|}
\hline \multirow[t]{4}{*}{ Atom } & \multirow[t]{4}{*}{$\mathrm{IP}_{\mathrm{HF}^{\mathrm{a}}}{ }^{\mathbf{a}}$} & \multirow[t]{4}{*}{${ }^{I} P_{D F}-I_{H F}{ }^{a)}$} & \multirow[t]{4}{*}{$\mathbf{I P}_{\exp }-\mathbf{I P}_{\mathbf{D F}}{ }^{\mathbf{b})}$} & \multicolumn{5}{|l|}{ Correlation c) } \\
\hline & & & & \multicolumn{2}{|l|}{ without SIC } & \multirow{2}{*}{\multicolumn{2}{|c|}{$\frac{\text { with SIC }}{\text { frozen core }}$}} & \multirow{3}{*}{$\begin{array}{l}\left.\operatorname{relax}^{3, h}\right) \\
\text { SPP }\end{array}$} \\
\hline & & & & \multirow[t]{2}{*}{ frozen core ${ }^{d}$ ) } & \multirow[t]{2}{*}{ relax $a, e)$} & & & \\
\hline & & & & & & $\left.P Z^{f}\right)$ & SPP g) & \\
\hline \multirow[t]{2}{*}{$\mathbf{K}$} & 147.2 & 0.5 & 11.8 & 175 & 16.9 & 5.5 & 4.2 & 3.8 \\
\hline & & & & 17.6 & 16.9 & 5.5 & 4.2 & 3.8 \\
\hline \multirow[t]{2}{*}{$\mathbf{R b}$} & 1375 & 2.0 & 14.0 & 17.6 & 16.8 & 6.1 & 4.6 & 4.1 \\
\hline & & & & 18.0 & 17.1 & 6.3 & 4.8 & 4.3 \\
\hline \multirow[t]{2}{*}{ Cs } & 123.2 & 4.7 & 15.2 & 17.0 & 16.1 & 6.1 & 4.6 & 4.1 \\
\hline & & & & 17.8 & 16.8 & 6.7 & 5.0 & 4.5 \\
\hline \multirow[t]{2}{*}{$\mathrm{Cu}$} & 2355 & 5.7 & 42.7 & 30.8 & 27.7 & 15.5 & 12.1 & 10.6 \\
\hline & & & & 31.7 & 28.4 & 16.3 & 12.8 & 11.0 \\
\hline \multirow[t]{2}{*}{$\mathrm{Ag}$} & 217.2 & 15.7 & 455 & 31.1 & 27.9 & 16.6 & 12.7 & 11.1 \\
\hline & & & & 33.8 & 29.9 & 18.8 & 14.4 & 12.4 \\
\hline \multirow[t]{2}{*}{$A u$} & 217.8 & 64.6 & 56.6 & 32.6 & 28.9 & 18.1 & 13.8 & 11.9 \\
\hline & & & & 41.6 & 35.1 & 25.5 & 19.4 & 16.1 \\
\hline \multirow[t]{2}{*}{$\mathrm{Ca}^{+}$} & 415.6 & 1.2 & 19.5 & 23.6 & 22.6 & 9.0 & 6.9 & 6.4 \\
\hline & & & & 23.7 & 22.7 & 9.1 & 7.0 & 6.5 \\
\hline \multirow[t]{2}{*}{$\mathrm{Sr}^{+}$} & 377.7 & 6.1 & 21.6 & 23.7 & 22.4 & 9.8 & 7.4 & 6.8 \\
\hline & & & & 24.1 & 22.8 & 10.1 & 7.7 & 7.0 \\
\hline \multirow[t]{2}{*}{$\mathrm{Ba}^{+}$} & 333.0 & 11.1 & 23.5 & 22.9 & 215 & 9.8 & 7.4 & 6.7 \\
\hline & & & & 23.9 & 22.3 & 10.6 & 8.0 & 7.2 \\
\hline \multirow[t]{2}{*}{$\mathrm{Zn}^{+}$} & 6085 & 10.3 & 41.4 & 35.8 & 32.9 & 18.2 & 14.3 & 12.8 \\
\hline & & & & 36.6 & 33.5 & 18.9 & 14.9 & 13.3 \\
\hline \multirow[t]{2}{*}{$\mathrm{Cd}^{+}$} & 544.8 & 26.3 & 50.2 & 36.6 & 33.3 & 20.0 & 15.5 & 13.8 \\
\hline & & & & 38.9 & 35.1 & 22.0 & 17.0 & 15.0 \\
\hline \multirow{2}{*}{$\mathrm{Hg}^{+}$} & 530.4 & 99.4 & 59.5 & 37.9 & 34.1 & 21.6 & 16.5 & 16.6 \\
\hline & & & & 45.5 & 39.6 & 28.0 & 21.3 & 18.3 \\
\hline
\end{tabular}

a) Reiasation taken into account. b) Experimental values from ref. [10].

c) The upper value is obtained $w$ ith HF densities, the lower one with DF densities.

d) Eq. (3). e) Difference of $E_{c}$ values, eq. (1). I) eq. (5).

g) Eq. (4). h) Difference of $E_{c}$ values, eq. (2).

$E_{\mathrm{c}}$ was obtained numerically (Simpson's rule) by using the grid of points given by RELAMC. The computing time was negligible compared to that of the HF or DF calculation (0.1\%).

In table 1 we present our computed values together with values deduced from experiment [10]. We compare our correlation energy contributions to the ionization potential with differences between the experimental and DF values. (We assume that the relativistic effects not included in RELAMC do not change our results significantly.) The LSD values with SPP SIC are systematically too low (they are $\approx 30 \%$ : between $26 \%$ for $\mathrm{Cu}$ and $33 \%$ for $\mathrm{Ca}^{+}$).
The values obtained with the scaled ("relativistic") density functional are not shown in table 1 because they differ by at most 0.1 mhartree from the non-relativistic ones. We explain this fact by the small portion of the valence density in the inner core region. On the other hand, use of relativistic instead of non-relativistic orbitals increases the contribution of the correlation energy by up to $26 \%$ ( 4 mhartree for $\mathrm{Au}$ ). This effect is larger for the transition than for the alkali and alkaline earth elements.

The effect of relaxation of the core orbitals after ionization is opposite to the previous one: a lowering of $10 \%$ is observed for the group IA and IB atoms, 
while it is slightly larger for the group IIA and IIB ones (up to 20\%, 3 mhartree. for $\mathrm{Au}$ ).

The PZ SIC increases the correlation contributions by $\approx 30 \%$ with respect to SPP SIC. The values with SIC are systematically too low. For the transition elements the LSD values are too low, even without SIC. A subtractive correction, as provided by SIC, cannot remedy this defect. Therefore we conclude that further improvement within the density-functional method requires a better description for the Coulomb hole.

We would like to thank Dr. J. Snijders for the stimulating discussions which were at the origin of the present work. We have also benefited from the profound knowledge of Dr. J.P. Desclaux. The authors wish to thank the Fonds der Chemischen Industrie and the Deutsche Forschungsgemeinschaft for financial support.

\section{References}

[1] J. Migdalek and W.E. Baylis,Can. J. Phys. 60 (1982) 1317.

[2] J.P. Desclaux, Computer Phys. Commun. 9 (1975) 31.

[3] S.H. Vosko, L. Wilk and M. Nusair, Can. J. Phys. 58 (1980) 1200.

[4] M.V. Ramana and A.K. Rajagopal, Phys. Rev. A24 (1981) 1689.

[5] U.V. Barth and L. Hedin, J. Phys. C5 (1972) 1629.

[6] J.P. Perdew and A. Zunger, Phys. Rev. B23 (1981) 5048.

[7] 11. Stoll, C.M. Pavlidou and H. Preuss, Theoret. Chim. Acta 49 (1978) 143.

[8] A. Savin, U. WVedig, H. Stoll and H. Preuss, Chem. Phy s. Letters 92 (1982) 503.

[9] A. Savin, M. Dolg, H. Stoll and H. Preuss, to be published.

[10] C.E. Moore, Atomic Energy Levels, NBS Circular 467, (National Bureau of Standards, Washington, 1949). 